\title{
ORIGINAL RESEARCH \\ Dural Tears in Spinal Burst Fractures: Predictable MR Imaging Findings
}

\begin{abstract}
I.S. Lee
H.J. Kim

J.S. Lee

S.-J. Kim

Y.J. Jeong

D.K. Kim

T.-Y. Moon

BACKGROUND AND PURPOSE: The diagnosis of traumatic spinal dural tears is difficult to establish. The purpose of this study was to determine the reliable MR imaging findings suggesting dural tears in spinal burst fractures.

MATERIALS AND METHODS: We retrospectively reviewed spine MR images of 21 patients with dural tears (study group) and 33 patients without dural tears (control group), all of whom had spinal burst fractures. The following MR imaging features were compared between the 2 groups: the interpedicular distance, the angle of the retropulsed segments, the ratio of the central canal diameter, the presence or absence of laminar fractures, the degree of laminar fractures, and the extent of epidural hemorrhage.
\end{abstract}

RESULTS: The mean values of the grade of the laminar fracture, the interpedicular distance, the ratio of the central canal diameter, the angle of the retropulsed segment, and the extent of epidural hemorrhage in the study and control groups were as follows: 1.77 and $0.86(P=.034), 28.7$ and $26 \mathrm{~mm}$ $(P=.02), 0.37$ and $0.58(P=.008), 112^{\circ}$ and $128^{\circ}(P=.05)$, and 2.37 and $1.4(P=.11)$, respectively. The ratio of the central canal diameter was the most reliable factor suggesting dural tears compared with other factors.

CONCLUSIONS: Dural tears are likely when there are MR imaging findings of laminar fracture of more than grade 1, the interpedicular distance is $>28 \mathrm{~mm}$, the central canal ratio is $<0.46$, and the acute angle of the retropulsed segment is $<135^{\circ}$.
B urst fracture of the thoracolumbar spine is defined as a failure of at least the anterior and middle columns of a vertebral segment because of axial compression, usually with some flexion. ${ }^{1}$ In the report of Keenen et al, ${ }^{2}$ a retrospective review of patients with spinal fractures revealed a $7.7 \%$ incidence of dural tears in surgically treated patients. The diagnosis of traumatic spinal dural tears is important because defects in the dura can acutely entrap nerve roots. In addition, dural tears increase the risk of meningitis, which may allow a dural laceration to remain open indefinitely and may lead to the development of a posttraumatic meningocele, which in itself can act as a chronic focus for nerve root entrapment. ${ }^{3}$ However, dural tears are very difficult to diagnose clinically or radiologically before surgery.

Until now, dural tears have traditionally been diagnosed by conventional myelography. However, myelography may not be performed unless the patient can tolerate the procedure without altering the neurologic status during the examination. ${ }^{3}$ Also, myelography is an invasive technique and can cause major complications such as infection or nerve injury, though these problems are very uncommon. Recently, evaluation of most spinal lesions has been performed with MR imaging because it can directly depict spinal fractures, paraspinal and vertebral soft-tissue edema, and ligamentous injuries. ${ }^{4-6}$ However, it is nearly impossible to visualize directly an accom-

Received April 21, 2008; accepted after revision July 1.

From the Medical Research Institute (I.S.L., H.J.K., J.S.L., S.-J.K., Y.J.J., D.K.K., T.-Y.M.), and Departments of Radiology (I.S.L., H.J.K., Y.J.J., D.K.K., T.-Y.M.), Orthopaedic Surgery (J.S.L.), and Nuclear Medicine (S.-J.K.), College of Medicine, Pusan National University, Busan, Republic of Korea.

Please address correspondence to Hak Jin Kim, MD, Department of Radiology, Pusan National University School of Medicine, 1-10 Ami-Dong, Seo-Gu, Busan 602-739, Korea; e-mail: hakjink@pusan.ac.kr

indicates article with supplemental on-line tables.

DOI 10.3174/ajnr.A1273 panying small dural tear $<1 \mathrm{~cm}$ on MR images. MR myelography (MRM) using a very heavily T2-weighted sequence can produce an effect similar to that of conventional myelography. ${ }^{7,8}$ However, MRM is not the method generally used at some institutions and is also not included in routine spinal trauma protocols at some hospitals. There have been no reports regarding dural tears in spinal burst fractures by using only T1- and T2-weighted images. Therefore, the purpose of the current study was to investigate indirect MR imaging findings on T1-and T2-weighted images that could suggest dural tears in patients with spinal burst fractures.

\section{Materials and Methods}

\section{Patients}

We analyzed the MR images of 21 patients ( 13 men and 8 women; mean age, 41.7 years; range, 22-76 years) with dural tears (study group) caused by spinal burst fractures and surgically confirmed between January 2005 and December 2006. Thirty-three patients with surgically proved spinal burst fractures (20 males and 13 females; mean age, 48.7 years; range, 17-77 years) without dural tears were retrospectively reviewed as a control group. Spinal burst fractures occurred between T12 and L5 in both patient groups. Patients with combined disk herniation or infectious conditions were excluded. We reviewed the operative reports in the medical charts to determine the size of the dural tears. The study was approved by our institutional review board. However, informed patient consent was not required for this retrospective study.

\section{MR Imaging Protocol}

MR imaging of the spine was performed by using a 1.5T system (Magnetom Vision or Sonata; Siemens, Erlangen, Germany) and a spinal surface or phased-array coil.

T1-weighted spin-echo images were obtained in the sagittal plane (TR/TE: mean, 549/14 ms; range, 363-752/10-23 ms; section thick- 

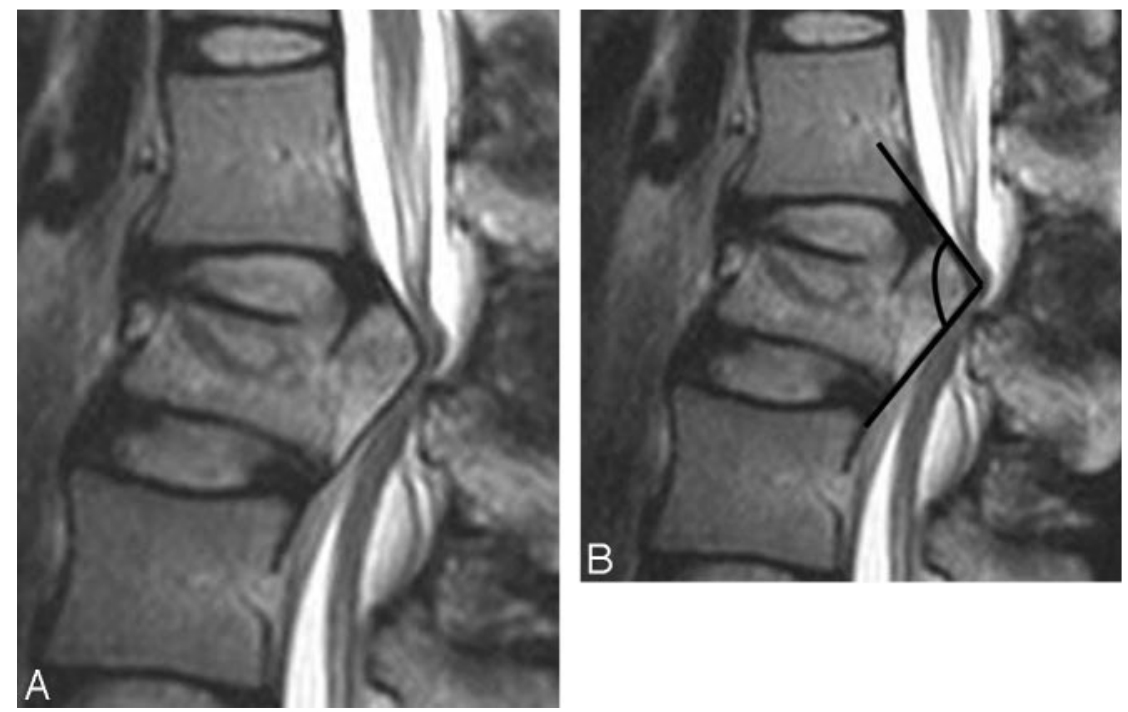

Fig 1. Measurement of the angle of the retropulsed segment in the spinal burst fracture. $A$, This was assessed in the portion having the most acute angle on the sagittal plane. $B$, We obtained an angle formed by crossing 2 lines drawn according to the cortical line of the retropulsed segment.

ness: mean, $4.13 \mathrm{~mm}$; range, $4-5 \mathrm{~mm}$; FOV: mean, $263 \times 263 \mathrm{~mm}$; range, $150-370 \times 150-370 \mathrm{~mm}$; matrix size: mean, $392 \times 205$; range, $256-512 \times 101-256 ; 2$ or 3 acquisitions) and in the transverse plane (TR/TE: mean, 659/13 ms; range, 450-1510/10-20 ms; section thickness: mean, $4.65 \mathrm{~mm}$; range, 3-6 mm; FOV: mean, $159 \times 159 \mathrm{~mm}$; range, $120-310 \times 120-310 \mathrm{~mm}$; matrix size: mean, $353 \times 196$; range, $256-512 \times 123-256 ; 2$ or 3 acquisitions). T2-weighted fast spin-echo images were obtained in the sagittal plane (TR/TE: mean, 3094.22/ $112.30 \mathrm{~ms}$; range, 1350-4000/97-127 ms; section thickness: mean, $4.13 \mathrm{~mm}$; range, $4-5 \mathrm{~mm}$; FOV: mean, $275 \times 275 \mathrm{~mm}$; range, $240-$ $370 \times 240-370 \mathrm{~mm}$; matrix size: mean, $580 \times 236$; range, $256-$ $1024 \times 200-358 ; 2$ or 3 acquisitions) and in the transverse plane (TR/TE: mean, 4522/115 ms; range, 2740-6200/90-147 ms; section thickness: mean, $4.65 \mathrm{~mm}$; range, 3-6 mm; FOV: mean, $150 \times 150$ $\mathrm{mm}$; range, $110-250 \times 110-250 \mathrm{~mm}$; matrix size, mean, $370 \times 208$; range, $256-512 \times 168-358 ; 2$ or 3 acquisitions).

In 30 patients (study group, 16 patients; control group, 14 patients), T1-weighted gadolinium-enhanced images were obtained in the sagittal plane (TR/TE: mean, 534/17 ms; range, 432-796/13-29 ms; section thickness: mean, $4.11 \mathrm{~mm}$; range, $4-5 \mathrm{~mm}$; FOV: mean, $252 \times 252 \mathrm{~mm}$; range, $130-300 \times 130-300 \mathrm{~mm}$; matrix size: mean, $313 \times 194$; range, $256-512 \times 104-256$; 2 or 3 acquisitions) with fat suppression and in the transverse plane (TR/TE: mean, 726/20 ms; range, 471-969/11-27 ms; section thickness: mean, $4.38 \mathrm{~mm}$; range, 4-6 mm; FOV: mean, $153 \times 153 \mathrm{~mm}$; range, $130-260 \times 130-260$ mm; matrix size: mean, $256 \times 195$; range, $256 \times 179-230$; 2 or 3 acquisitions).

\section{Analysis of the Spinal MR Images}

The MR images were reviewed by 2 experienced musculoskeletal radiologists by consensus. First, we investigated the presence or absence of a pseudomeningocele, suggesting a nerve root sleeve or arachnoid membrane tear on axial T2-weighted MR images. Second, we evaluated the fractured vertebrae by using the following: interpedicular (IP) distance, angle of the retropulsed segment of the vertebral body, ratio of the central canal diameter, the presence or absence of a laminar fracture, and the degree of the laminar fracture on T1- and T2weighted MR images. Third, we also evaluated the extent of epidural hemorrhage.

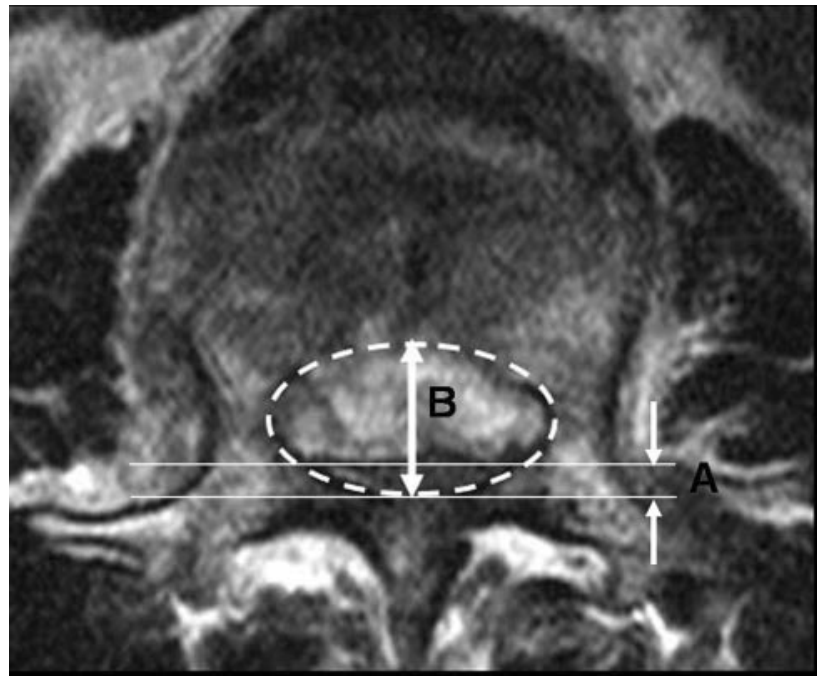

Fig 2. The ratio of central canal diameter. The central canal diameter was measured with each AP diameter of the normal central canal $(B)$ and the narrowest portion of central canal (A) drawn by an imaginary line at the burst fracture level. The ratio of central canal diameter reduction was calculated dividing $B$ by $A$ as seen on the axial image.

The IP distance was determined at the widest point of the fracture level in the axial plane. The angle of the retropulsed vertebral body was measured in the portion having the most acute angle in the sagittal plane (Fig 1). The central canal diameter was measured with the anteroposterior (AP) diameter of the normal central canal (B) and the narrowest portion of the central canal (A) drawn by an imaginary line at the burst fracture level. The ratio of central canal diameter reduction was calculated by dividing B by A as seen on axial image (Fig 2). The imaginary line was drawn in an up-and-down fashion symmetrically in a semicircle along the inner cortical line of the lamina and pedicle of the vertebrae. In the central canal of the oval shape obtained with the previously described method, we measured the AP diameter. For optimal accuracy, 2 radiologists independently measured the ratio of the central canal diameter and then measured it again several days later. The final results were obtained by consensus. We also evaluated the presence or absence and the degree of the laminar fracture. If there was a laminar fracture, the degree was classified as follows: 0 , 

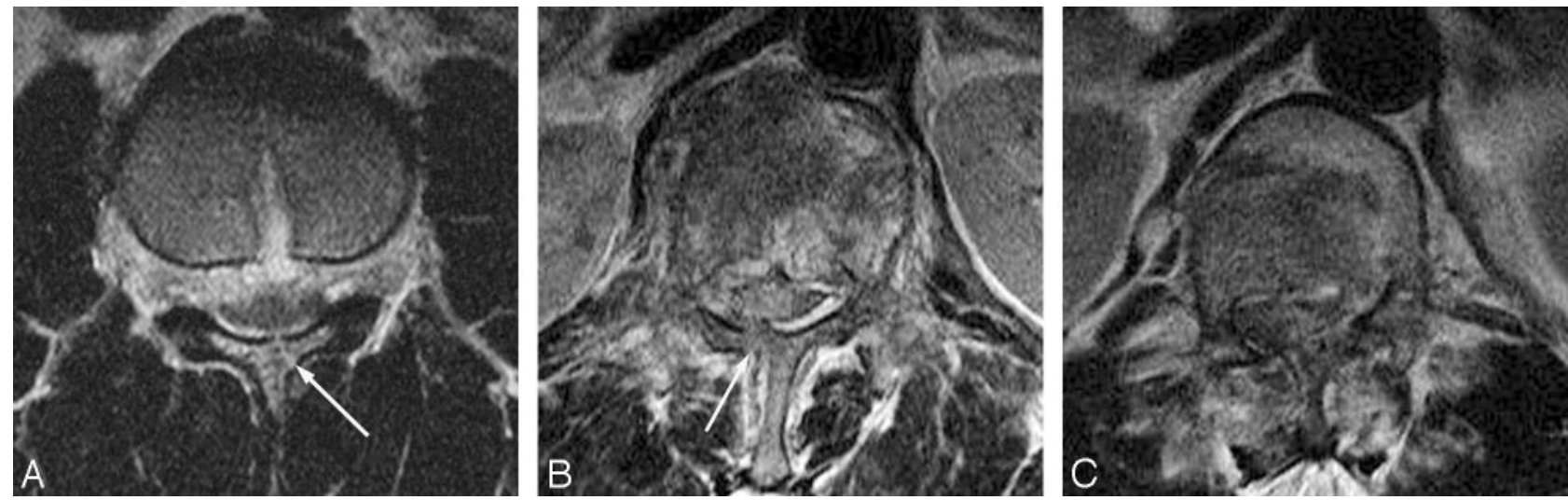

Fig 3. The grades of laminar fractures. The degree is classified according to the following: 0 , no fracture; 1 , fracture without gap $(A)$; 2 , fracture with gap $(B)$; and 3 , displaced fracture $(C)$

\begin{tabular}{|c|c|c|c|}
\hline & Study Group & Control Group & $P$ value \\
\hline IP distance & $28.7(24-39) \mathrm{mm}$ & $26(18-34) \mathrm{mm}$ & .02 \\
\hline Angle & $112^{\circ}\left(64-180^{\circ}\right)$ & $128^{\circ}\left(66-180^{\circ}\right)$ & .05 \\
\hline Ratio of central canal & $0.37(0.13-0.65)$ & $0.58(0.21-0.86)$ & .008 \\
\hline Lamina fracture & $1.77(0-3)$ & $0.86(0-2)$ & .003 \\
\hline Extent of EDH & $2.4(0-6)$ & $1.4(0-4)$ & .11 \\
\hline
\end{tabular}

Note:-EDH indicates the number of vertebral bodies involved in the epidural hemorrhage.

no fracture; 1, fracture without a gap; 2, fracture with gap; and 3, displaced fracture (Fig 3). We defined the higher grade of the laminar fracture as the more unstable fracture. The extent of epidural hemorrhage was represented by the number of vertebral bodies within the epidural hemorrhage.

\section{Statistical Analysis}

We used the Mann-Whitney $U$ test and the $\chi^{2}$ test to determine the significant differences between the 2 patient groups in regard to each factor, by using the Statistical Package for the Social Sciences for Windows 10.0 (SPSS, Chicago, Ill). In particular, regarding the central canal diameter ratio, $\kappa$ statistics were applied to determine intra- and interobserver agreement. In addition, stepwise logistic regression was used to determine the most meaningful of 5 imaging findings. To identify the optimal threshold value of the factors suggesting a dural tear, we performed receiver operating characteristic (ROC) curve analysis. Standard error (SE), 95\% confidence interval (CI), and area under curve were also calculated. The previously mentioned methods were implemented by using commercially available software (MedCalc Software, Mariakerke, Belgium). A $P$ value $<.05$ was considered to indicate a statistically significant difference.

\section{Results}

The results of each MR imaging finding in the study and control groups are summarized in on-line Tables 1 and 2. Twentytwo vertebrae of 21 patients in the study group and 35 vertebrae of 33 patients in the control group showed burst fractures, which were surgically confirmed. In the operative fields, dural tears in the study group were confirmed in the posterior aspect. The extent of the tear ranged from approximately $5 \mathrm{~mm}$ to $4 \mathrm{~cm}$ (Table 1 ). In the study group, 8, 4, 4, 2, and 1 patients had burst fractures at the L1, L2, L3, T12, and L5 levels, respectively. In the control group, 10, 6, 6, 9, 3, and 1

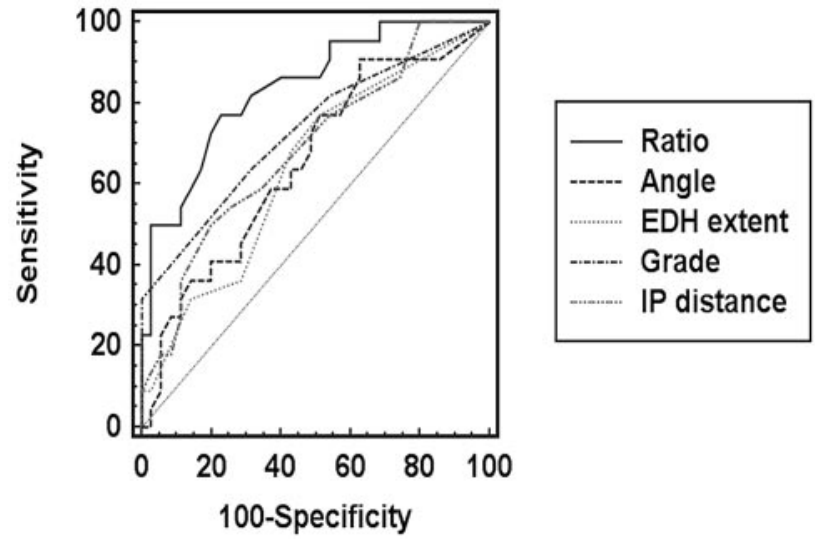

Fig 4. The ROC curve in each parameter. This graph shows that the ratio is the most significant among the 5 parameters used to predict a dural tear. Ratio indicates the ratio of the central canal diameter; Angle, the angle of the retropulsed segment; EDH extent, the number of vertebral bodies involved in the epidural hemorrhage; Grade, the grade of laminar fracture; IP distance, the distance between pedicles.

patients had burst fractures at the L1, L2, L3, T12, L4, and L5 levels, respectively. Pseudomeningoceles were not revealed on MR images in any patient in either group. The mean values of each parameter and the statistical significance in the study and control groups are summarized in Table 1. We obtained both interobserver $(\kappa=0.86)$ and intraobserver (each $\kappa=0.79$ and $\kappa=0.82)$ agreement regarding the ratio of the central canal diameter. Not only the degree of instability $(P=.003)$ but also the presence or absence of a laminar fracture $(P=.034)$ showed a statistically significant relationship to the dural tear.

In the ROC curve analysis, the ratio of a central canal diameter $<0.5$ (area under the ROC curve, 0.8 ; 95\% CI, 0.7-0.9; SE, $0.1 ; P=.000)$, angle $<135^{\circ}$ in the retropulsed segment at the burst fracture level (area under the curve, 0.7; 95\% CI, 0.5-0.8; SE, $0.1 ; P=.03)$, an IP distance exceeding $28 \mathrm{~mm}$ (area under the curve, $0.7 ; 95 \% \mathrm{CI}, 0.6-0.8 ; \mathrm{SE}, 0.1 ; P=.01$ ), and a laminar fracture exceeding grade 1 (area under the curve, $0.7 ; 95 \%$ CI, $0.6-0.8$; SE, $0.1 ; P=.000$ ) could be used as threshold values to suggest a dural tear in a spinal burst fracture (Fig 4). According to logistic regression, the ratio of the central canal diameter was the most reliable factor suggesting a dural tear compared with other factors (coefficient, 2.4; 95\% CI, 3.1-37.3; SE, 0.6; odds ratio, 10.7; and $P=.000$ ). However, the sensitivities of each parameter were relatively low (laminar 
fracture, $82 \%$; IP distance, $55 \%$; the ratio of the central canal diameter, 77\%; and the angle of retropulsed segment, $86 \%$ ). Therefore, simultaneous consideration of all 4 parameters might be necessary for interpretation of a dural tear, with a concomitant increase in the sensitivity and the accuracy.

\section{Discussion}

According to our results, the IP distance, the central canal diameter ratio, the angle of the retropulsed segment, and the laminar fractures were significant parameters representing dural tears in spinal burst fractures. The wider the IP distance, the greater was the incidence of dural tears $(P=.02)$. Miller et $\mathrm{al}^{9}$ also documented dural lacerations in patients with thoracolumbar burst fractures associated with pedicle separation. According to the study of Tacar et al, ${ }^{10}$ the ranges of the transverse diameters (IP distances) of each segmental level in adult men and women are as follows; L1, 26.25-27.17/ 5.03-25.70; L2, 26.91-27.81/25.60-26.37; L3, 28.01-28.93/26.80-27.60; L4, 29.29-30.52/28.14-29.20; and L5, 33.27-34.75/31.8033.31 in men/women. Generally, the IP distance becomes wider at the lower lumbar level. Therefore, the IP distance at the level of a burst fracture should be compared with the IP distances at both the upper and lower lumbar levels.

According to the study of Aydinli et al, ${ }^{11}$ a $20 \%$ increase in the IP distance results in a 79\% probability of greenstick laminar fractures. Many investigators have found that a burst fracture with an associated laminar fracture was 100\% sensitive and $74 \%$ specific for the presence of a dural tear. ${ }^{9,12}$ In particular, posterior dural lacerations are always associated with laminar fractures and are caused by the posteriorly displaced dural sac being impaled on the sharp edges of the laminar fracture. ${ }^{13}$ Therefore, patients with neurologic deficits and a laminar fracture associated with a lumbar burst fracture have an increased risk of a dural laceration and also the possibility of entrapment of neural elements. ${ }^{12}$ However, it is not always possible to predict preoperatively whether a patient without neurologic deficits but with a greenstick laminar fracture has dural tears and/or entrapment of nerve roots. ${ }^{14}$ In the present study, laminar fractures were significantly associated with dural tears. In addition, the higher degree of laminar fractures, the higher was the rate of dural tears.

The bony elements of the middle column in the vertebral compression are retropulsed into the spinal canal; these bone and disk fragments impact the neural structures, displace the dural sac in the posterior direction, and narrow the spinal canal. ${ }^{15}$ Silvestro et al ${ }^{16}$ reported that severe encroachment of the spinal canal was frequently associated with dural tears. Although they found that the degree of spinal canal narrowing was not statistically significant, the ratio of the central canal diameter was the most meaningful factor in our results. A central canal narrowed approximately less than one half compared with the normal cross-sectional diameter was highly associated with a dural tear. Moreover, our results showed that more acute angles of the retropulsed column had a higher incidence of dural tear.

Burst fractures are the result of vertical compressive and flexion forces. ${ }^{1,17}$ Aside from iatrogenic etiologies, many dural tears occur as a result of trauma. ${ }^{18}$ Although the clinical significance of dural lacerations caused by burst fractures remains to be assessed, dural lacerations can result in diffusion of the blood within the subdural space, leak of CSF leading to pseudomeningocele, trapping of herniated nerve roots, and delayed scars involving the neural structures..$^{9,12,19-24}$ Therefore, a number of observations suggest that knowledge of the actual presence of dural tears in patients with spinal fractures could represent a relevant adjunct to the rationale for optimal management. ${ }^{9,14,16}$ Identification of dural tears through imaging techniques or surgical approach is also important for preventing further neurologic injury and promoting neurologic recovery during the treatment of spinal burst fractures. If radiologists mention the possibility of a dural tear in the report of spine MR imaging, the surgeons will make an effort to identify the presence or absence of a true dural tear, even of very small size around fracture sites. Therefore, the frequency of missing a dural tear of small size may be decreased in the operative field.

Although identification of a dural tear before surgery is important, direct visualization of a dural tear is often difficult. Unfortunately, many previous reports have not provided clearly suggestive radiologic findings of dural tears. ${ }^{9,12,16} \mathrm{MR}$ imaging has excellent resolution of soft-tissue structures and fluid and, as such, is very sensitive for CSF accumulation and pseudomeningoceles. ${ }^{18}$ A comparison study showed that MR imaging was more accurate for detecting suggested CSF fistula than was surgery. ${ }^{25}$ However, in this study, because pseudomeningocele or CSF leakage was not detected on T2-weighted images, we could not confirm the CSF leakage caused by the dural tear. Moreover, relatively large dural tears of 3-4 cm were not detected on MR imaging. We thought that direct discontinuity of the dural wall or nerve root herniation into the epidural space was not seen because severe encroachment of the spinal canal and an epidural hematoma disturbed evaluation of the dura itself or the exact location of nerve roots.

Contrast myelography with injection of contrast material into the thecal sac has been used with some success and is sometimes needed in cases of acute trauma. ${ }^{18}$ However, it may not be performed because the required patient manipulation can produce or aggravate neurologic deficits. ${ }^{26,27}$ In addition, although it may suggest nerve roots outside the dura and the desirability of exposing the epidural space to look for dural rents and herniation, it is now obsolete because it lacks crosssectional features and anatomic superposition. ${ }^{28,29}$ Use of MRM was first reported by Krudy, ${ }^{30}$ who used heavily T2weighted fast spin-echo imaging with fat suppression. Enhancement of the CSF signal intensity and suppression of the background signal intensity in MRM make this method ideally suited for demonstrating posttraumatic root avulsion diverticula. Obtaining a single composite MRM allows a noninvasive cost-effective demonstration of both large and small diverticula. ${ }^{31}$ However, in the study of Gasparotti et al, ${ }^{32}$ compared with cervical CT myelography, 3D MRM showed $89 \%$ sensitivity, $95 \%$ specificity, and $92 \%$ diagnostic accuracy in identifying nerve root avulsion. Moreover, 3D MRM is also subject to motion artifacts despite its short examination time. Therefore, direct visualization of a dural tear by using MRM in spinal trauma is not complete.

Until now, there have been no specific or sensitive imaging techniques to reveal a small dural tear or direct CSF leakage in a burst spinal fracture. Therefore, our intention was to determine the indirect findings of dural tears on spine MR imaging. 
On the basis of previous reports, ${ }^{12,14,16}$ we determined $5 \mathrm{MR}$ findings suggesting dural tears and assessed the significance of those findings. Although Pau et $\mathrm{al}^{14}$ had also reported that encroachment of the spinal canal, separation of the pedicles, and laminar fractures were related to dural lacerations subsequent to spinal burst fractures, they concluded that none of these factors were significant statistically. However, in our study, 4 findings (except the extent of epidural hemorrhage) were statistically significant in identifying dural tears.

Our study had several limitations. First, the number of patients included was small. Second, the exact measurement of the central canal diameter ratio was difficult, especially in cases of markedly unstable fractures. However, we obtained excellent interobserver $(\kappa=0.86)$ and intraobserver (each $\kappa=0.79$ and $\kappa=0.82$ ) agreements. Third, because this was a retrospective review and we did not perform MRM, we could not identify how often pseudomeningoceles by dural tears were detected on MRM.

In conclusion, radiologists might suggest the high possibility of a dural tear when there are MR imaging findings of the central canal severely narrowed to less than one half by displaced bone fragments with a more acute angle, more unstable laminar fracture, and wider IP distance at the burst fracture level.

\section{References}

1. Denis $F$. The three column spine and its significance in the classification of acute thoracolumbar spinal injuries. Spine 1983;8:817-31

2. Keenen TL, Antony J, Benson DR. Dural tears associated with lumbar burst fractures. J Orthop Trauma 1990;4:243-45

3. Morris RE, Hasso AN, Thompson JR, et al. Traumatic dural tears: CT diagnosis using metrizamide. Radiology 1984;152:443-46

4. Marciello MA, Flanders AE, Herbison GJ, et al. Magnetic resonance imaging related to neurologic outcome in cervical spinal cord injury. Arch Phys Med Rehabil 1993;74:940-46

5. Lee RR. MR imaging and cervical spine injury. Radiology 1996;201:617-18

6. Flanders AE, Schaefer DM, Doan HT, et al. Acute cervical spine trauma: correlation of MR imaging findings with degree of neurologic deficit. Radiology 1990;177:25-33

7. Ross JS, Modic MT. Current assessment of spinal degenerative disease with magnetic resonance imaging. Clin Orthop Relat Res 1992;68-81

8. Jensen MC, Kelly AP, Brant-Zawadzki MN. MRI of degenerative disease of the lumbar spine. Magn Reson Q 1994;10:173-90

9. Miller CA, Dewey RC, Hunt WE. Impaction fracture of the lumbar vertebrae with dural tear. J Neurosurg 1980;53:765-71
10. Tacar O, Demirant A, Nas K, et al. Morphology of the lumbar spinal canal in normal adult Turks. Yonsei Med J 2003;44:679-85

11. Aydinli U, Karaeminogullari O, Tiskaya K, et al. Dural tears in lumbar burst fractures with greenstick lamina fractures. Spine 2001;26:E410-15

12. Cammisa FP Jr, Eismont FJ, Green BA. Dural laceration occurring with burst fractures and associated laminar fractures. J Bone Joint Surg Am 1989;71:1044-52

13. Pickett J, Blumenkopf B. Dural lacerations and thoracolumbar fractures. J Spinal Disord 1989;2:99-103

14. Pau A, Silvestro C, Carta F. Can lacerations of the thoraco-lumbar dura be predicted on the basis of radiological patterns of the spinal fractures? Acto Neurochir (Wien) 1994;129:186-87

15. Denis F. Spinal instability as defined by the three-column spine concept in acute spinal trauma. Clin Orthop Relat Res 1984;65-76

16. Silvestro C, Francaviglia N, Bragazzi R, et al. On the predictive value of radiological signs for the presence of dural lacerations related to fractures of the lower thoracic or lumbar spine. J Spinal Disord 1991;4:49-53

17. Carl AL, Matsumoto M, Whalen JT. Anterior dural laceration caused by thoracolumbar and lumbar burst fracture. J Spinal Disord 2000;13:399-403

18. Bosacco SJ, Gardner MJ, Guille JT. Evaluation and treatment of dural tears in lumbar spine surgery: a review. Clin Orthop Relat Res 2001;389:238-47

19. Jelsma RK, Kirsch PT, Jelsma LF, et al. Surgical treatment of thoracolumbar fractures. Surg Neurol 1982;18:156-66

20. Jelsma RK, Kirsch PT, Rice JF, et al. The radiographic description of thoracolumbar fractures. Surg Neurol 1982;18:230-36

21. Osaka K, Handa H, Watanabe $H$. Traumatic intrathoracic meningocele (traumatic subarachnoid-pleural fistula). Surg Neurol 1981;15:137-40

22. Rawlings CE 3rd, Nashold BS Jr. Traumatic extradural spinal cysts: a case report and review of the literature. Br J Neurosurg 1989;3:403-08

23. Schmidek HH, Gomes FB, Seligson D, et al. Management of acute unstable thoracolumbar (T-11-L-1) fractures with and without neurological deficit. Neurosurgery 1980;7:30-35

24. Shuman WP, Rogers JV, Sickler ME, et al. Thoracolumbar burst fractures: CT dimensions of the spinal canal relative to postsurgical improvement. AJR Am J Roentgenol 1985;145:337-41

25. Johnson DB, Brennan P, Toland J, et al. Magnetic resonance imaging in the evaluation of cerebrospinal fluid fistulae. Clin Radiol 1996;51:837-41

26. Leo JS, Bergeron RT, Kricheff II, et al. Metrizamide myelography for cervical spinal cord injuries. Radiology 1978;129:707-11

27. Post MJ, Green BA. The use of computed tomography in spinal trauma. Radiol Clin North Am 1983;21:327-75

28. Brant-Zawadzki M, Jeffrey RB Jr, Minagi H, et al. High resolution CT of thoracolumbar fractures. AJR Am J Roentgenol 1982;138:699-704

29. Albayram S, Kilic F, Ozer H, et al Gadolinium-enhanced MR cisternography to evaluate dural leaks in intracranial hypotension syndrome. AJNR Am J Neuroradiol 2008;29:116-21

30. Krudy AG. MR myelography using heavily T2-weighted fast spin-echo pulse sequences with fat presaturation. AJR Am J Roentgenol 1992;159:1315-20

31. el Gammal TA, Crews CE. MR myelography of the cervical spine. Radiographics 1996;16:77-88

32. Gasparotti R, Ferraresi S, Pinelli L, et al. Three-dimensional MR myelography of traumatic injuries of the brachial plexus. AJNR Am J Neuroradiol 1997;18: $1733-42$ 